\title{
Characterization of spannability of functions
}

\author{
Farhad Hüsseinov
}

Bilkent University, Faculty of Economics, Administratrive and Social Sciences, 06533 Bilkent, Ankara, Turkey

\begin{abstract}
Possibility of representation of a value of convexification of function at the given point as a convex combination of the values of function (spannability) is studied. Spannability of functions turned out to be important in different fields e.g. in the study of quasi-cores of monetary economies with nonconvex preferences (mathematical economics), in the theory of relaxation of variation problems (variational calculus). Apparently, Shapley and Shubik (Econometrica, 1966, 34, 805-827) were first to discuss it.
\end{abstract}

JEL classification: CGO

\section{Introduction}

This article is devoted to the study of spannability. A necessary and sufficient condition for spannability of a lower semicontinuous function with a graph not containing a line is obtained (Theorem 1). Also the case of a function with graph containing lines is studied (Theorem 2). Results obtained here are of a general nature and can potentially be used in order to study the relaxation of convexity conditions, for instance in the framework of general equilibrium among others.

Spannability of a function means that the graph of its convexification (i.e. the greatest lower semicontinuous convex function not exceeding the function) is contained in the convex hull of the function's graph. (For an explicit definition see Section 2.) Apparently, at least in the context of economic theory (cores and competitive equilibria) Shapley and Shubik (1966) were first to discuss the spannability. Spannability of utility functions of traders turned out to be important in their study of quasi-cores of monetary economies with nonconvex preferences. Shapley and Shubik (1966, p. 806) noted that "a condition of 'spannability' ... 
helps restore some of the regularity that is lost when concavity [of utility functions or convexity of preferences] is abandoned, and which seems potentially useful beyond the immediate context of cores and competitive equilibria" and also ( $p$. 819) "[spannability of functions] appears to be quite fundamental to any investigation of the relaxation of convexity conditions." This last insight proved to be quite right. Specifically, spannability turned out to be quite fundamental in the study of the important problem of relaxation of variational problems (see e.g. Ekeland and Temam, 1976, and more earlier references there). Here the spannability of integrands is of great importance for the construction of relaxation of variational problems.

Shapley and Shubik (1966, theorem 3) gave some conditions on a function guaranteeing its spannability. Another sufficient condition ( $\alpha$-coerciveness for $a>1$; see Section 2 for definition) was obtained by Ekeland and Temam (1976, p. 280 , lemma 3.3 ) in connection with the study of relaxation of multidimensional variational problems. This result was further strengthened by Hüsseinov (1988) and somewhat later by Griewank and Rabier (1990, p. 693-697) to the case of 1 -coercive functions. As the simple example after Corollary 3 below shows, the power $\alpha=1$ is sharp.

Shapley and Shubik assumed identical tastes for all involved agents represented by a nonconcave utility function $U$, that is, they allow for nonconvex preferences. They called $U$ spannable if its negative $-U$ is spannable according to the definition above. Clearly everey concave function is spannable according to their definition. An economy in their paper is given by a specification of the set of agents $N=\{1,2, \cdots, n\}$, initial endowments of agents and of utility function $U$. Using these data they constructed a finite game in characteristic form with characteristic function $v: 2^{N} \rightarrow R$. Strong (weak) $\epsilon$-core is defined as a set of payoff vectors $\alpha=\left(\alpha_{1}, \alpha_{2}, \cdots, \alpha_{n}\right)$ satisfying

$$
\sum_{i \in S} \alpha_{i} \geq v(S)-\epsilon, \text { for all } S \subset N,
$$

$\left(\sum_{i \in S} \alpha_{i} \geq v(S)-s \epsilon\right.$ for all $S \subset N$, where $\left.s=\# S\right)$. Evidently, every strong $\epsilon$-core allocation is a weak $\epsilon$-core allocation. Shapley and Shubic showed non-emptiness of both $\epsilon$-cores for sufficiently large replicas of the given economy. They discussed sociological interpretations of both kinds of cores and sociological consequences of these results. Non-emptiness of the weak $\epsilon$-core is shown almost at no condition on function $U$. However, the proof of existence of the strong $\epsilon$-core requires spannability and some differentiability property of $U$. Since these conditions are just sufficient for the existence of the strong $\epsilon$-core one might think that the same result is true under assumptions of a quite different nature on $U$. However, this is not true. Actually any set of sufficient assumptions should involve some variant of spannability (perhaps, some sort of approximate spannability), i.e. it is an almost necessary ingredient of any set of sufficient conditions. 
The author is not familiar with any result on spannability except those referred to above. All of them turn out to be corollaries of Theorem 1. It should be noted that the case studied in Theorem 2 sharply differs from the case considered in Theorem 1. In this case the characterization includes an explicit condition on the extreme points of some restriction of function, whereas in the first case the analog of that property is established on account of such a banal condition as lower semicontinuity. As simple examples show, this 'lack' of Theorem 2 is inherent for the case of a function with nontrivial lineality space of its epigraph (for definitions see Section 2). Specifically, the main result of this paper (Theorem 1) could be used to clarify if a utility function is spannable, and therefore by the use of Shapley and Shubik's result discussed above, to decide if there exists a strong $\epsilon$-core in an economy involving this utility function. Indeed, since the domain of a utility function $U$ in Shapley- Shubik's model is the positive orthant, or more generally, one just assumes this domain (consumption set) to be bounded from below, it follows that the epigraph of convexification of $-U$ does not contain lines (see the last paragraph of the next section). Then one of the two essential conditions of Theorem 1 is satisfied almost at no condition on $U$. Note that the condition of lower semicontinuity and affinely boundedness from below involved in this theorem is quite technical. Therefore the unique essential condition of Theorem 1 to be verified in order to determine if a given utility function is spannable is Assumption 1 formulated before Proposition 2 below. This seems to be a solvable task in many cases. Simple example can be constructed to illustrate this point.

\section{Notations and definitions}

We will freely use the usual notations and concepts from convex analysis. All of them can be found in Rockafellar (1970). We present reminders of some of them here and define some less common concepts. $\bar{R}=R \cup\{-\infty, \infty\}$ the extended real line; $R^{n}$-n-dimensional real coordinate space; $\operatorname{dom} f$, epi $f$ and $\operatorname{gr} f$ are respectively, the domain, epigraph and graph of function $f ; f^{* *}$ the greatest lower semicontinuous convex function not exceeding $f$. Recall that $\operatorname{dom} f=\{x \in$ $\left.R^{n}: f(x)<\infty\right\}$ and epi $f=\left\{(x, \alpha) \in R^{n} \times R: a \geq f(x)\right\}$. Notation $f^{* *}$ is common, its origin due to the well-known fact that $f^{* *}$ is the second Legendre transformation of $f$. For the set $A \subset R^{n}$ we will denote by $A$ and $\partial A$ respectively its interior and boundary. A point $a$ of a convex set $A$ is called an extreme point if it cannot be represented as a convex combination of two points from $A$ different from $a$. An exposed point is an extreme point through which there is a supporting hyperplane which contains no other points of $A$. An extreme ray is a face which is half-line. Recall that $A^{\prime} \subset A$ is called a face if every line segment in $A$ with a relative interior point in $A^{\prime}$ has both end-points in $A^{\prime}$. 
A lineality space of a convex set $A$ is defined as an intersection of asymptotic cones of $A$ and $-A$. Specifically, it is a subspace consisting of vectors $\boldsymbol{x}$ such that $A+\lambda x \subset A$ for all $\lambda \in R$. A function $f$ is said to be affinely bounded from below if there exists an affine function not exceeding $f$ everywere. For an affine subspace $M \subset R^{n}$, its subset $M_{0}$ will be called embracing if the convex hull of $M_{0}$ coincides with $M$. Recall that function $f: R^{n} \rightarrow \bar{R}$ satisfying the condition $\lim _{\|x\| \rightarrow \infty} f(x) /\|x\|^{\alpha}(\alpha>0)$ is called $\alpha$-coercive. If $\lim _{\|x\| \rightarrow \infty} f(x)=\infty$ function $f$ is simply called coercive.

For a subspace $L \subset R^{n}$ by $L^{\perp}$ and $\operatorname{Pr}_{L}$ will be denoted its orthogonal complement and orthogonal projection operator to $L$, respectively.

The following definition is central for this paper.

Definition 1. A function $f: R^{n} \rightarrow \bar{R}$ is called spannable if for any point $x \in R^{n}$ there exist points $x_{1}, \ldots, x_{m} \in R^{n}$ and nonnegative numbers $\lambda_{1}, \ldots, \lambda_{m}$ with $\lambda_{1}+\ldots+\lambda_{m}=1$ such that

$$
x=\sum_{i=1}^{m} \lambda_{i} x_{i} \text { and } f^{* *}(x)=\sum_{i=1}^{m} \lambda_{i} f\left(x_{i}\right),
$$

i.e. the graph of function $f^{* *}$ is contained in the convex hull of the graph of function $f$.

It can be easily shown that in Definition 1 the integer $m$ can be restricted to $n+1$.

The convex envelope conv $f$ of $f$ is defined as the greatest convex function not exceeding $f$ everywerc. It is well known (see Rockafellar and Tyrrel, 1970, p. 36), and can be easily seen. that

$$
(\operatorname{conv} f)(x)=\inf \sum_{i=1}^{p} \lambda_{i} f\left(x_{i}\right),
$$

where the infimum is taken over all convex combinations $\sum_{i=1}^{p} \lambda_{i} x_{i}=x$ with an arbitrary $p$ (provided $f$ does not assume the value $-\infty$, so that the summation is unambiguous). It is asumed that $\lambda_{i} f\left(x_{i}\right)=0$ when $\lambda_{i}=0$ and $f\left(x_{i}\right)=+\infty$.

Clearly, the convexification $f^{* *}$ of $f$ is the lower semicontinuous extension of conv $f$, that is, the greatest lower semicontinuous function majorized by conv $f$.

In the definition of spannability above we could replace $f^{* *}$ by conv $f$ and get a weaker concept of spannability. The relative interiors of domains of the convexification $f^{* *}$ and the convex envelope conv $f$ coincide and these functions coincide on this common relative interior of their domain. Therefore for points from ri $\operatorname{dom} f^{* *}=$ ri doni conv $f$ the two definitions of spannability do not differ. It should be noted that actually under assumptions of all results on spannability referred to above and the results of the present paper the two concepts of spannability coincide. 
Any function $f: R^{n} \rightarrow \bar{R}$ defined on a subset $A$ of $R^{n}$ extended to $R^{n}$ as $+\infty$ out of $A$ will be treated as a function defined on $R^{n}$. So the above definition of spannability equally applies to the functions defined on subsets of $K^{n}$.

\section{Spannability}

The following propositions are stages of a proof of the main theorem (Theorem 1) on spannability.

Proposition 1. Let a function $f: R^{n} \rightarrow \bar{R}_{+}$be brunded away from zero on a closed half-space $E \subset R^{n}$ and $f^{* *}(x)$ tend to infinity for $x \in E,\|x\| \rightarrow \infty$. Then $f^{* * *}$ is strictly positive on open half-space $\stackrel{\circ}{E}$.

Proof. Let $M_{t}(t \in R)$ be a family of the hyporplanes parallel to $\partial E$ and such that $t=\operatorname{dist}\left(M_{t}, \partial E\right)$ for $M_{t}$ not contained in $E$ and $t=-\operatorname{dist}\left(M_{t}, \partial E\right)$ for $M_{t}$ contained in $E$. It is easily seen that a function of a scalar variable $h$ defined as $h(t)=\inf \left\{f(x) \mid x \in M_{t}\right\}$ is a nonnegative function tending to infinity for $t \rightarrow-\infty$ and is bounded from zero on $(-\infty, 0]$. Let us define also a function of the scalar variable $\tilde{h}(t)=\inf \left\{f^{* *}(x) \mid x \in M_{t}\right\}$ and show that

$$
\tilde{h}(t)=h^{* *}(t) \text {. }
$$

It can be easily checked that $\tilde{h}$ is a convex, lower semicontinuous function not exceeding $h$. Therefore, $\tilde{h} \leq h^{* *}$. We must show that inverse inequality is also true. Fix $t_{0} \in R$ and $\epsilon>0$. Let $x_{t_{0}} \in M_{t_{0}}$ be an arbitrary point. Since

$$
f^{* *}\left(x_{t_{0}}\right)=\inf \left\{\sum_{i=1}^{m} \lambda_{i} f\left(x_{i}\right): \lambda_{i} \geq 0, \sum_{i=1}^{m} \lambda_{i}=1, \sum \lambda_{i} x_{i}=x_{t_{0}}, m \in N\right\}
$$

then there exist points $x_{\mathrm{i}}(i=1, \ldots, \mathrm{m})$ and nonnegative numbers $\lambda_{i}(i=1, \ldots, m)$ such that $\sum_{i=1}^{m} \lambda_{i} x_{i}=x_{t_{0}}$ and $\sum_{i=1}^{m} \lambda_{i} f\left(x_{i}\right)<f^{* *}\left(x_{t_{0}}\right)+\epsilon$.

Denote by $t_{i}(i=1, \ldots, m)$ a number for which $x_{i} \in M_{t_{i}}(i=1, \ldots, m)$. Then $h\left(t_{i}\right) \leq f\left(x_{i}\right)(i=1, \ldots, m)$ and therefore

$$
\sum_{i=1}^{m} \lambda_{i} h\left(t_{i}\right)<f^{* *}\left(x_{t_{0}}\right)+\epsilon .
$$

Besides, since $\sum_{i=1}^{m} \lambda_{i} x_{i}=x_{t_{0}}$, then clearly

$$
\sum_{i=1}^{m} \lambda_{i} t_{i}=t_{0}
$$

the last two relations show that $h^{* *}\left(t_{0}\right) \leq f^{* *}\left(x_{t_{0}}\right)$. Since $x_{t_{0}}$ is an arbitrary point of hyperplane $M_{t_{0}}$ then we have $h^{* *}\left(t_{0}\right) \leq \tilde{h}\left(t_{0}\right)$. 
Coincidence (1) prompts that $h^{* *}(t)$ tends to $+\infty$ for $t \rightarrow-\infty$. Thus $h$ is a nonnegative function such that $h(t) \geq c>0$ for $t \leq 0$ and $h^{* *}(t) \rightarrow+\infty$ for $t \rightarrow-\infty$. It easily follows from these properties of $h$ that

$$
h^{* *}(t)>0 \text { for } t<0 \text {. }
$$

Since $h^{* *}(t)=\tilde{h}(t)=\inf \left\{f^{* *}(x) \mid x \in M_{t}\right\}$ then

$$
f^{* *}(x)>0 \text { for } x \in \stackrel{\circ}{E}=\bigcup_{t<0} M_{t} \text {. }
$$

The following condition on function $f: R^{n} \rightarrow \bar{R}$ will be referred to as Assumption 1 (see also Theorem 1 below): the graph of $f$ contains an unbounded subset of any nonvertical extreme ray of the epigraph of $f^{* *}$.

Proposition 2. Let $f: R^{n} \rightarrow \bar{R}$ be a lower semicontinuous nonnegative function satisfying Assumption 1. Then the set of zeros of function $f^{* *}$ coincides with the convex hull of the set of zeros of function $f$.

Proof. Denote by $N$ and $N^{* *}$, respectively, the sets of zeros of functions $f$ and $f^{* *}$. Let $x_{0} \in R^{n}$ be an exposed point of the convex closed set $N^{* *}$. Let $M$ be a hyperplane support to the convex closed set $N^{* *}$ at $x_{0}$ such that $M \cap N^{* *}=\left\{x_{0}\right\}$. Denote by $E$ and $E^{\prime}$ closed half-spaces defined by $M$ and such that $E^{\prime}$ contains $N^{* *}$. Clearly $E \cap N^{* *}=\left\{x_{0}\right\}$ and then $f^{* *}(x)>0$ for $x \in E \backslash\left\{x_{0}\right\}$. We will show that $f^{* *}(x) \rightarrow+\infty$ for $x \in E,\|x\| \rightarrow \infty$. Suppose on the contrary there exists a sequence $x_{k} \in \mathrm{E}(k=1,2, \ldots)$, such that $\left\|x_{k}\right\| \rightarrow \infty$ and $f^{* *}(x) \leq C$ $(k=1,2 \ldots)$ for some number $C$. Consider the sequence $x_{k} /\left\|x_{k}\right\|(k=1,2, \ldots)$. Without loss of generality, we can suppose that it is converging. Denote by $\bar{x}$ its limit. It is easily seen that the function $f^{* *}$ vanishes along the ray $d$ issuing from the point $x_{0}$ in the direction of the vextor $\bar{x}$. Clearly $d$ is contained in $E$. But this contradicts that $E \cap N^{* *}=\left\{x_{0}\right\}$.

If we suppose now $f\left(x_{0}\right)>0$ then from the lower semicontinuity of function $f$ and coercivity of $f^{* *}$, as proved above, on $E$ it follows that $f$ is bounded from zero on $E_{t}$ for sufficiently small $t>0$. Here by $E_{t}$ is denoted that of two closed half-spaces defined by the hyperplane $M_{t}$, which contains $E$. It is easily seen also that $f^{* *}$ is coercive on $E_{t}$. Then by Proposition $1, f^{* *}$ is strictly positive on $E_{t}$. But obviously, $x_{0} \in E_{1}$ and hence $f^{* *}\left(x_{0}\right)>0$, which contradicts $f^{* *}\left(x_{0}\right)=0$.

Since the set of exposed points of closed convex set $N^{* *}$ is dense in the set of extreme points (see Rockafellar, 1970, theorem 18.6) and set $N$ is closed (because $f$ is lower semicontinuous) then any extreme point of $N^{* *}$ is contained in $N$.

Let now $e$ be an arbitrary extreme ray of the set $N^{* *}$. Then clearly $e$ is an extreme ray of epi $f^{* *}$ and then by Assumption 1 contains an: unbounded sequence from $N$. Clearly the origin of $e$, as an extreme point of $N^{* *}$, also belongs to $N$. Therefore the whole ray $e$ is contained in $\operatorname{co} N$. 
Proposition 3. Let a function $f: R^{n} \rightarrow \bar{R}$ be as in Theorem 1 (lower semicontinuous, affinely bounded from below and satisfying Assumption I). Then graph $f \supset$ ex(epi $f$ ); in other words, $f$ coincides with $f^{* *}$ on the projection on $R^{n}$ of the set of extreme points of the epigraph of function $f^{* *}$.

Proof. Suppose first that $x_{0} \in R^{n}$ is the projection of exposed point $z_{0}=\left(x_{0}\right.$, $\left.f^{* *}\left(x_{0}\right)\right)$ of epi $f^{* *}$. Since $z_{0}$ is an exposed point there exits a hyperplane $\Gamma$ support to epi $f^{* *}$ and such that (epi $f^{* *}$ ) $\cap \Gamma=\left\{z_{0}\right\}$. It is easily seen that $\Gamma$ is not vertical. Indeed if $\Gamma$ was vertical, then it would contain the vertical ray $e=\left\{\left(x_{0}, f^{* *}\left(x_{0}\right)+\alpha\right): \alpha \geq 0\right\}$. Since $e \in$ epi $f^{* *}$, then $e \in\left(\right.$ epi $\left.f^{* *}\right) \cap \Gamma$, which contradicts (epi $\left.f^{* *}\right) \cap \Gamma=\left\{z_{0}\right\}$. Since $\Gamma$ is nonvertical, it is a graph of some linear function $l$. Consider a function $g=f-l$. Obviously, $g^{* *}=f^{* *}-l$, and $x_{0}$ is a unique zero of function $g^{* *}$. Then by Pruposition $2, g\left(x_{0}\right)=0$, i.e. $f\left(x_{0}\right)=l\left(x_{0}\right)=f^{* *}\left(x_{0}\right)$.

Suppose now $z_{0}=\left(x_{0}, f^{* *}\left(x_{0}\right)\right)$ is an arbitrary extreme point of epi $f^{* * *}$. Then by the above cited theorem 18.6 from Rockafellar (1970), there exists a sequence $\left.\left\{z_{\mathrm{k}}=x_{k}, f^{* *}\left(x_{k}\right)\right)\right\}$ of exposed points of epi $f^{* *}$ converging to $z_{0}$. As is proved above, $f\left(x_{k}\right)=f^{* *}\left(x_{k}\right),(k=1,2 \ldots)$. This together with the lower semicontinuity of $f$ and $f^{* *}$ and convexity of $f^{* *}$ implies $f\left(x_{0}\right)=\liminf _{k} f\left(x_{k}\right)$ $=\lim \inf _{k} \mathrm{f}^{* *}\left(x_{k}\right)=f^{* *}\left(x_{0}\right)$, whence $f\left(x_{0}\right)=f^{* *}\left(x_{0}\right)$.

Theorem 1. Let $f: R^{n} \rightarrow \bar{R}$ be a lower semicontinuous affinely bounded from below function such that epi $f^{* *}$ does not contain lines. Then $f$ is spannable if and only if its graph contains an unbounded subset of any nonvertical extreme ray of the epigraph of $f^{* *}$.

Proof. Necessity. Suppose the assumption of Theorem 1 is not satisfied, i.e. there exists a nonvertical extreme ray $\bar{e}$ of epi $f^{* *}$ such that a set $\bar{e} \cap$ graph $f$ is bounded. Denote by $e$ the unit vector with the direction of ray $\bar{e}$. Let $\bar{z}$ be the vertex of ray $\bar{e}$ and $z_{0} \in \bar{e}$ such that half-line $\left\{\left(z_{0}-e\right)+\alpha e: \alpha \geq 0\right\}$ is contained in $\bar{e}$, and does not intersect the graph of function $f$ :

$$
\left\{\left(z_{0}-e\right)+\alpha e: \alpha \geq 0\right\} \cap \text { graph } f=\emptyset \text {. }
$$

We assert that the point $z_{0}=\left(x_{0}, f^{* *}\left(x_{0}\right)\right)$ has no representation (1). Indeed, if such a representation exists, since the coefficients $\lambda_{k}(k=1, \ldots, m)$ are strictly positive, the point $z_{0}$ is a relative interior point of the polytope $P=\operatorname{co}\left\{\left(x_{k}\right.\right.$, $\left.\left.f\left(x_{k}\right)\right): k=1, \ldots, m\right)$. Since $\bar{e}$ is a face of epi $f^{* *}$, then by theorem 18.1 from Rockafellar (1970), polytope $P$ is contained in $\bar{e}$. In particular, the points $z_{k}$ $(k=1, \ldots, m)$ are contained in $\bar{e}$. This, together with (3) gives $z_{k} \in\left[\bar{z}, z_{0}-e\right]$, $k=1, \ldots, m$. But in this case $P$ also is contained in the convex set $\left[\bar{z}, z_{0}-e\right]$, whence $z_{0} \notin P$, which contradicts $z_{0} \in$ ri $P$.

Sufficiency: If the assumption of Theorem 1 is satisfied, then clearly any nonvertical extreme ray of epi $f^{* *}$ is contained in $\operatorname{co}(g r a p h f)$. By Proposition 3, 
graph $f$ contains all extreme points of epi $f^{* *}$. In particular, we have from that that any vertical extreme ray of epi $f^{* *}$ is contained in epi $f$. Since epi $f^{* *}$ does not contain lines, then by virtue of theorem 18.5 from Rockafellar (1970), we obtain

$$
\operatorname{co}\left(\text { graph } f \cup\left(\text { vertical extreme rays of epi } f^{* *}\right)\right) \supset \text { epi } f^{* *},
$$

and then, in particular,

co(epi $f \cup\left(\right.$ vertical extreme rays of epi $\left.\left.f^{* *}\right)\right) \supset$ graph $f^{* *}$.

Thus, for each point $x_{0} \in \operatorname{dom} f^{* *}$, the point $\left(x_{0}, f^{* *}\left(x_{0}\right)\right) \in \operatorname{co}($ epi $f)$. Therefore, there exist points $x_{1}, \ldots, x_{m} \in R^{n}$ and numbers $\lambda_{1}, \ldots, \lambda_{m}>0$, $\sum_{k=1}^{m} \lambda_{k}=1$ and $\alpha_{1}, \ldots, \alpha_{m} \geq 0$ such that

$$
\left(x_{0}, f^{* *}\left(x_{0}\right)\right)=\sum_{k=1}^{m} \lambda_{k}\left(x_{k}, f\left(x_{k}\right)+\alpha_{k}\right) .
$$

If some of the numbers $\alpha_{k}$ are strictly positive, replacing them by zero in the right-hand side, we obtain that $x_{0}=\sum_{k=1}^{m} \lambda_{k} x_{k}$ and $f^{* *}\left(x_{0}\right)>\sum_{k=1}^{m} \lambda_{k} f\left(x_{k}\right)$, which contradicts the definition of $f^{* *}$. Let now $\Gamma$ be a hyperplane support to epi $f^{* *}$ at the point $\left(x_{0}, f^{* *}\left(x_{0}\right)\right)$. Then clearly all points $\left(x_{k}, f\left(x_{k}\right)\right)(k=$ $1, \ldots, m)$ are contained in $\Gamma$. Since $\operatorname{dim} \Gamma=n$, by Caratheodory's theorc 1 (e.g. Rockafellar, 1970, p. 155), the point $\left(x_{0}, f^{* *}\left(x_{0}\right)\right)$ is also a convex combination of at most $n+1$ of points $\left(x_{k}, f\left(x_{k}\right)\right)(k=1, \ldots, m)$. Therefore, we can suppose that $m \leq n+1$. Thus,

$$
\left(x_{0}, f^{* *}\left(x_{n}\right)\right)=\sum_{k=1}^{m} \lambda_{k}\left(x_{k}, f\left(x_{k}\right)\right),
$$

where $m \leq n+1$ and $\lambda_{\mathrm{k}}>0, k=1, \ldots, m, \sum_{k=1}^{m} \lambda_{k}=1$ or equivalently

$$
\begin{aligned}
& x_{0}=\sum_{k=1}^{m} \lambda_{k} x_{k}, \\
& f^{* *}\left(x_{0}\right)=\sum_{k=1}^{m} \lambda_{k} f\left(x_{k}\right)
\end{aligned}
$$

and the required representation has a place. Theorem 1 is proved.

Corollary 1. let $f: R^{n} \rightarrow \bar{R}$ be a lower semicontinuous, affinely bounded from below function, such that dom $f \subset R^{n}$ does not contain lines. The $f$ is spannable if and only if its graph contains an unbounded subset of any nonvertical extreme ray of the epigraph of $f^{* *}$.

Proof. It is easily seen that epi $f^{* *}$ does not contain a nonvertical line, because if it did contain such a line, then projection onto $R^{n}$ of that line should be line contained in dom $f$. Obviously, epi $f^{* *}$ also does not contain vertical lines. 
The following corollary is a translation of theorem $\mathbf{3}$ from Shapley and Shubik (1966) from concave framework into convex. We will preface it with some necessary definitions. A function $f: R_{+}^{n} \rightarrow \bar{R}$ is called suplinear, if for every linear function $l$ with negative coefficients the difference $f-l$ has a finite lower bound. A function $f$ will be called strictly decreasing, if for each $x, y \in R_{+}^{n}$ such that $y-x \in R_{+}^{n}, y-x \neq 0, f(x)<f(y)$.

Corollary 2. If $f: R_{+}^{n} \rightarrow \vec{R}$ is continuous suplinear and strictly decreasing, then $f$ is spannable (Shapley and Shubik 1966).

Proof. Let us show that the graph of convexification $f^{* *}$ does not contain any rays. To this end, let us first show that $f^{* *}$ is strictly decreasing. Fix points $x_{0}$, $x_{1} \in R_{+}^{n}$ such that $z=x_{1}-x_{0} \in R_{+}^{n} \backslash\{0\}$. Let $p$ be any positive normalized vector in $R^{n}$. Denote $D=\left\{x \in R^{n}: p x \leq p x_{0}+1\right\}$ and $\delta_{1}=\inf \{f(x)-f(x+z): x \in D\}$ which is positive, because $D$ is compact and $f$ is continuous.

Let $x_{1}, \ldots, x_{\mathrm{m}} \in R_{+}^{n}$ be arbitrary points and $\lambda_{1}, \ldots, \lambda_{m}$ be arbitrary nonnegative numbers such that $\sum_{k=1}^{m} \lambda_{k}=1$ and $\sum_{k=1}^{m} \lambda_{\mathrm{k}} x_{k}=x_{0}$. Denote $I_{0}=\left\{k: x_{k} \in D\right\}$ and $I_{1}=\{1, \ldots, m\} \backslash I_{0}$. Then

$$
\sum_{k \in I_{0}} \lambda_{k} \geq \delta_{0}
$$

for a positive number $\delta_{0}=1 /\left[2\left(p x_{0}+1\right)\right]$. Indeed, if $\Sigma_{k \in I_{0}} \lambda_{k}<\delta_{0}$, then

$$
\begin{aligned}
p \cdot\left(\sum_{k \in I_{1}} \lambda_{k} x_{k}\right) & =\sum_{k \in I_{1}} \lambda_{k} p \cdot x_{k}>\sum_{k \in I_{1}} \lambda_{k}\left(p \cdot x_{0}+1\right)>\left(1-\delta_{0}\right)\left(p \cdot x_{0}+1\right) \\
& >p \cdot x_{0} .
\end{aligned}
$$

Therefore

$$
p \cdot x_{0}=p \cdot\left(\sum_{k \in I_{1}} \lambda_{k} x_{k}\right)=p \cdot \sum_{k \in I_{0}} \lambda_{k} x_{k}+p \cdot \sum_{k \in I_{l}} \lambda_{k} x_{k}>p \cdot x_{0},
$$

and thereby (7) is proved.

Obviously,

$$
\sum_{k \in I_{1}} \lambda_{k}\left(x_{k}+z\right)=x_{0}+z=x_{1} \text {. }
$$

Then using (7) we will have

$$
\begin{aligned}
\sum_{k \in I_{1}} \lambda_{k} f\left(x_{k}+z\right) & =\sum_{k \in I_{0}} \lambda_{k} f\left(x_{k}+z\right)+\sum_{k \in I_{1}} \lambda_{k} f\left(x_{k}+z\right) \\
& <\sum_{k \in I_{0}} \lambda_{k}\left(f\left(x_{k}\right)-\delta_{1}\right)+\sum_{k \in I_{1}} \lambda_{k} f\left(x_{k}\right) \\
& \leq \sum_{k=1}^{m} \lambda_{k} f\left(x_{k}\right)-\delta_{0} \delta_{1},
\end{aligned}
$$


whence,

$$
f^{* *}\left(x_{1}\right) \leq f^{* *}\left(x_{0}\right)-\delta_{0} \delta_{1}<f^{* *}\left(x_{1}\right),
$$

Since $f^{* *}$ is nonincreasing, we have for $x_{0}=0$ and $x_{1} \in R_{+}^{N}, x_{1} \neq 0, f\left(x_{1}\right)<$ $f\left(x_{1} / 2\right) \leq f\left(x_{0}\right)$. Thus $f^{* *}$ is strictly increasing. Consequently, gr $f^{* *}$ may contain just downward rays. This is also impossible, because of suplinearity of $f^{* *}$, which immediately follows from suplinearity of $f$. Thus, gr $f^{* *}$ does not contain any ray. Therefore the convex closed set epi $f^{* *}$ has no nonvertical extreme rays. By Corollary $1, f$ is spannable.

Remark. In the proof of Corollary 2 we established that a function $f$ satisfying assumptions of Shapley-Shubik's theorem in addition to spannability has the following properties: convexification $f^{* *}$ is strictly decreasing and there is no ray in $R_{+}^{n}$ such that the restriction of $f^{* *}$ to that ray is affine.

Corollary 3. Let $f: R^{n} \rightarrow \bar{R}$ be a lower semicontinuous function such that

$$
\lim _{\|x\| \rightarrow x} \frac{f(x)}{\|x\|}=\infty .
$$

Then $f$ is spannable.

Proof. Cleariy $f$ is bounded from below and it is not difficult to show that convexification $f^{* *}$ also satisfies assumption (9). Therefore $\operatorname{gr} f^{* *}$ does not contain any ray and then epi $f^{* *}$ does not contain any nonvertical extreme ray. By Theorem 1 , function $f$ is spannable.

Corollary 3, in particular, contains Ekeland and Temam's lemma 3.3 (1976, p. 280), where instead of assumption (9) a more restrictive assumption $\lim _{\|x\| \rightarrow x}$ $f(x) /\left[\|x\|^{\alpha}\right]=\infty(\alpha>1)$ is adopted. As the following example show the growth condition (9) is sharp.

\section{Example 1.}

$$
f(x)=\left\{\begin{array}{cc}
2|x| & \text { if }|x| \leq 1 \\
|x|+1 & \text { if }|x|>1
\end{array}\right.
$$

Then $f^{* *}(x)=|x|$ and clearly $f$ is not spannable.

Theorem 2. Let $f: R^{n} \rightarrow \bar{R}$ be an affinely bounded from below fuiction with nontrivial lineality space $L$ of epi $f^{* *}$. Then $f$ is spannable if and only if projection $\operatorname{Pr}_{L} \perp\left(\mathrm{gr} f \cap\right.$ epi $\left.f^{* *}\right)$ contains an unbounded subset of any nonvertical extreme ray of epi $f^{* *} \cap L^{\perp}$ and moreover, for extreme point $\overline{\text { of }}$ the set epi $f^{* *} \cap L^{\perp} t^{\prime}$ e graph of $f$ contains an embracing subset of $\bar{z}+L$. 
Proof. Without loss of generality, we will assume that $f$ is nonnegative and the lineality space $L$ of epi $f^{* *}$ is contained in the horizontal hyperplane $R^{n} \times\{0\}$ in $R^{n+1}=R^{n} \times R$. Hyperplane $R^{n} \times\{0\}$, as above, will be identified with $R^{n}$, then $L$ will be a subspace of $R^{n}$.

We will assume also that $L$ is different from $R^{n}$ (the case $L=R^{n}$ is trivial).

Let $f$ be spannable and $\bar{z}$ be an extreme point of epi $f^{* *} \cap L^{\perp}$. Let $\Gamma$ be a hyperplane support to epi $f^{* *}$ at $\bar{z}$. Obviously, $\Gamma$ contains $\bar{z}+L$, moreover, $\bar{z}+L$ is a face of $\Gamma \cap$ epi $f^{* *}$. Since $f$ is spannable, then for an arbitrary point $z=\left(x_{0}\right.$, $\left.f^{* *}\left(x_{0}\right)\right)$ of $\bar{z}+L$ there exist points $z_{k}=\left(x_{k}, f\left(x_{k}\right)\right)(k=1,2, \ldots, m)$ and positive numbers $\lambda_{1}, \ldots, \lambda_{m}, \lambda_{1}+\cdots+\lambda_{m}=1$, such that $z=\lambda_{1} z_{1}$ $+\cdots+\lambda_{m} z_{m}$, (or $\left(x_{0}, f^{* *}\left(x_{0}\right)\right)=\lambda_{1}\left(x_{1}, f\left(x_{1}\right)\right)+\cdots+\lambda_{m}\left(x_{m}, f\left(x_{m}\right)\right)$. Since $z \in \bar{z}+L \subset \Gamma$ and all points $z_{1}, \ldots, z_{m}$ are placed above $\Gamma$, then $z_{1}, \ldots, z_{m} \in \Gamma$ and then $z_{1}, \ldots, z_{\mathrm{m}} \in \Gamma \cap$ epi $f^{* *}$. Moreover, since $\bar{z}+L$ is a face of $\Gamma \cap$ epi $f^{* *}$ and $z \in \bar{z}+L$ is a relative interior point of polytope $P=\operatorname{co}\left\{z_{1}, \ldots, z_{m}\right\}$, then $P \subset \bar{z}+L$ and then, in particular, $z_{1}, \ldots, z_{m} \in \bar{z}+L$. Thus for any point $z \in \bar{z}+L$ there are points $z_{1}, \ldots, z_{m} \in \bar{z}+\mathbf{L}$ such that $z \in \operatorname{co}\left\{z_{1}, \ldots, z_{m}\right\}$. Therefore the graph of function $f$ contains an embracing subset of $\bar{z}+L$.

Let us suppose now that $\overline{\mathrm{e}}$ is a nonvertical extreme ray of epi $f^{* *} \cap L^{\perp}$ not satisfying the assumption of Theorem 2, i.e. $\operatorname{Pr}_{L} \perp$ (gr $f \cap$ epi $f^{* *} ;, \bar{e}$ is bounded. Then there exists a segment $\left[z_{0}, \overline{z_{1}}\right] \subset \bar{e}$, where $\overline{z_{0}}$ is the vertex of $\bar{e}$, such that

$$
\operatorname{Pr}_{L} \perp\left(\text { gr } f \cap \text { epi } f^{* *}\right) \subset\left[\bar{z}_{0}, \bar{z}_{1}\right]
$$

Consider $\left(z_{1}+\bar{e}\right)+L$. Obviously, this set does not contain any point of the set gr $f \cap$ epi $f^{* *}$. Let $\Gamma^{\perp}$ be a hyperplane in $L^{\perp}$ support to epi $\left.f^{* *}\right|_{L^{\perp}}$ at $\overline{z_{1}}$ in $L^{\perp}$.

Obviously, $\Gamma^{\perp}$ contains $\bar{e}$. Therefore $\Gamma=\Gamma^{\perp}+L$ is a hyperplane in $R^{n+1}$ support to epi $f^{* *}$ and contains $\bar{e}+L$. Since $\bar{e}$ is an extreme ray of convex closed set $\Gamma^{\perp} \cap$ epi $\left.f^{* *}\right|_{L^{\perp}}$, then $\bar{e}+L$ is a face of $\Gamma \cap$ epi $f^{* *}$. Since we assumed function $f$ to be spannable, and a ray $\bar{e}$, in particular, a point $z_{0}=\bar{z}_{1}+e$ (e is a unit vector with direction $\bar{e}$ ) belong to $\mathrm{gr} f^{* *}$, it is a convex combination of points $z_{1}, \ldots, z_{\mathrm{m}} \in \operatorname{gr} \hat{f}$, i.e.

$$
z_{0}=\lambda_{1} z_{1}+\cdots+\lambda_{m} z_{m}, \lambda_{k}>0 k=1, \ldots, m, \lambda_{1}+\cdots+\lambda_{m}=1 .
$$

Then the relative interior of polytope $P=\operatorname{co}\left\{z_{1}, \cdots, z_{m}\right\}$ intersects $\bar{e}+L$.

By theorem 18.1 from Rockafellar (1970) polytope $P$ is contained in the face $\bar{e}+L$. It follows from (11) that at least one of the points $z_{1}, \ldots, z_{m}$ must belong to $z_{0}+\bar{e}+L$, which contradicts (10).

Sufficiency: suppose the assumptions of Theorem 2 are satisfied. First of all, by virtue of the second assumption (embracing), without loss of generality, we may assume that $f^{* *}$ coincides with $f$ on the set $\operatorname{Pr}_{R^{n}} \bar{z}+L$ for any extreme point $\bar{z}$ of the set epi $f^{* *} \cap L^{\perp}$. Thus $\operatorname{gr} f \cap L^{\perp}=\operatorname{gr}\left(f \hat{L}_{L_{0}^{\perp}}\right)\left(L_{0}^{\perp}=P_{R^{n}} L^{\perp}\right)$ contains all extreme points of epi $f^{* *} \cap L^{\perp}=$ epi $\left.f^{* *}\right|_{L_{0}^{+}}$and contains an unbounded subset 
of any extreme ray of epi( $\left.\left.f^{* *}\right|_{L_{0}^{1}}\right)$. Then as was shown in the proof of Theorem 1 ,

$$
\operatorname{co}\left(\left.\operatorname{grf}\right|_{L_{0}^{\perp}}\right) \supset \operatorname{epi}\left(\left.{ }^{* * *}\right|_{L_{0}^{\perp}}\right) .
$$

since the convex closed set epi $\left.f^{* *}\right|_{L_{0}^{1}}$ does not contain lines it has at least one extreme point $z_{0}$. Thus gr $f$ contains the affine space $\overline{z_{0}}+L$ parallel to $L$. From here and (11), we have

$$
\text { co grf } \supset\left(\text { epi } f^{* *} \cap L_{0}^{1}\right) \cup\left(\bar{z}_{0}+L\right) \text {. }
$$

Therefore co gr $f \supset$ epi $f^{* *}=\left(\right.$ epi $\left.f^{* *} \cap L^{\perp}\right)+L$.

\section{References}

Ekeland, I. and R. Temam, 1976, Convex analysis and variational problems (North-Holland, Amsterdam).

Griewank, A. and P.J. Rabier, 1990, On the smoothness of convex envelopes, Transactions of the American Mathematical Society 322, 691-709.

Hüsseinov, F.V., 1988, Ekeland's lemma and existence of regular minimizing sequences, Preprint 274 (Physics Institute of Academy of Sciences of Azerbaijan).

Rockafellar, R.T., 1970, Convex analysis (Princeton University Press, Princeton).

Shapley, L.S. and M. Shubik, 1966, Quasi-cores in a monetary economy with nonconvex preferences, Econometrica 34, 805-827. 